that montelukast appears to be equally effective in the low FeNO group suggest the either the current markers of eosinophilic lung disease are insufficiently sensitive to pick up low levels of leukotriene activation in the low FeNO group, or that montelukast has its antitussive activity by an alternative mechanism.

\section{S34 THE EFFECTS OF A NOVEL FORMULATION OF INHALED CROMOLYN SODIUM (PA101) IN IDIOPATHIC PULMONARY FIBROSIS AND CHRONIC COUGH: A RANDOMISED, CONTROLLED, PHASE 2 TRIAL}

${ }^{1}$ SS Brring, ${ }^{2} \mathrm{MS}$ Wijsenbeek, ${ }^{3} \mathrm{~S}$ Agrawal, ${ }^{4} \mathrm{JWK}$ van den Berg, ${ }^{5} \mathrm{H}$ Stone, ${ }^{6} \mathrm{TM}$ Maher, ${ }^{7} \mathrm{~A}$ Tutuncu, ${ }^{8} \mathrm{AH}$ Morice. 'Division of Asthma, Allergy and Lung Biology, King's College London, London, UK; ' ${ }^{2}$ Dept. Respiratory Medicine, Erasmus University Medical Centre, Rotterdam, The Netherlands; ${ }^{3}$ Dept. Respiratory Medicine, Glenfield Hospital, Leicester, UK; ${ }^{4}$ Dept. Pulmonology, Isala Hospital, Zwolle, Netherlands; ${ }^{5}$ Dept. Respiratory Medicine, Royal Stoke University Hospital, Stoke-on-Trent, UK; ${ }^{6}$ Royal Brompton Hospital and Fibrosis Research Group, National Heart and Lung Institute, Imperial College, London, UK; ${ }^{7}$ Patara Pharma, San Diego, California, US; ${ }^{8}$ Hull York Medical School, Castle Hill Hospital, Hull, UK

\subsection{6/thoraxjnl-2017-210983.40}

Introduction and Objectives Cough can be a debilitating symptom of idiopathic pulmonary fibrosis (IPF). The efficacy and safety of inhaled PA101, a novel formulation of cromolyn sodium was investigated in IPF patients with chronic cough in a multi-centre, randomised, double-blind, placebo-controlled, 2-cohort, 2-period, cross-over trial. To explore the mechanism of anti-tussive activity of PA101, a parallel study of similar design was conducted in patients with chronic idiopathic cough (CIC).

Methods Twenty-four participants with IPF and chronic cough were randomised to receive PA101 $(40 \mathrm{mg})$ or matching placebo three times a day for 2 weeks, followed by 2 weeks wash out, and then crossed over. The primary outcome measure was objective daytime cough frequency (from 24 hour acoustic recording, Leicester Cough Monitor) and secondary outcomes included subjective cough-specific quality of life (LCQ) assessed at Day 14. The primary efficacy analysis was based on a mixed effects model. In the CIC cohort, twentyeight participants were randomised in a study with similar design and endpoints.

Results In IPF patients, PA101 reduced daytime cough frequency by $31.1 \%$ at day 14 compared to placebo. Daytime cough frequency decreased from a mean(SD) of 55(55) to 39 (29) coughs/hour at day 14 with PA101 versus 51(37) to 52 (40) coughs/hour following placebo (figure 1). There was a significant correlation coefficient between change in cough severity (VAS score) and change in daytime cough frequency with PA101 at Day 14, $r=0.415 \quad(p=0.048)$. There was a trend towards improvement in LCQ score with PA101 compared to placebo; mean treatment difference 1.1, $\mathrm{p}=0.09$. PA101 was associated with an improvement in chest symptom and psychological domains of the KBILD-QOL $(p<0.05)$. In contrast, no treatment benefit for PA101 was observed in the CIC cohort; mean reduction of daytime cough frequency at Day 14 for PA101 adjusted for placebo was 6.2\%. PA101 was well tolerated in both cohorts and the incidence of adverse events was comparable between PA101 and placebo treatments.

Conclusions This study suggests that the mechanism of cough in IPF may be disease specific. Inhaled PA101 may be a treatment option for chronic cough in patients with IPF and warrants further investigation.

\section{S35 RANDOMISED CONTROL TRIAL QUANTIFYING THE EFFICACY OF LOW DOSE MORPHINE IN A RESPONDER GROUP OF PATIENTS WITH REFRACTORY CHRONIC COUGH}

'B Al-Sheklly, ${ }^{2}$ J Mitchell, 'B Issa, ${ }^{1} \mathrm{H}$ Badri, ${ }^{2} \mathrm{~S}$ Satia, ${ }^{2} \mathrm{~T}$ Collier, ${ }^{1} \mathrm{~S}$ Sen, ${ }^{1} \mathrm{~J}$ Webber Ford, ${ }^{1} \mathrm{D}$ Corfield, ${ }^{1} \mathrm{JA}$ Smith. ${ }^{1}$ University of Manchester, Manchester, UK; ${ }^{2}$ University Hospital South Manchester, Manchester, UK

\subsection{6/thoraxjnl-2017-210983.41}

Introduction and objectives The pathophysiology of chronic cough remains poorly understood and treatment options are limited. Morphine sulphate can improve quality of life in refractory chronic cough, ${ }^{1}$ but its mechanism of action and effect on objective cough frequency remain unknown. Here we report morphine's influence on cough frequency and patient reported outcomes in a group of refractory chronic cough patients who responded favourably to morphine in our tertiary clinic.

Methods We recruited 22 patients (mean age 61.7 years, 18 female, mean cough duration 14 years) taking low dose morphine for refractory chronic cough into a double blinded, placebo controlled, crossover study. Volunteers withdrew their morphine therapy and were randomised to receive morphine (5-10 mg BD slow release)/matched placebo during two treatment period (5-7 days duration) separated by a 5-7 day washout. At baseline and at the end of each treatment period we assessed 24 hour cough recordings using a semi-automated cough counter (vitaloJAK), cough severity (visual analogue

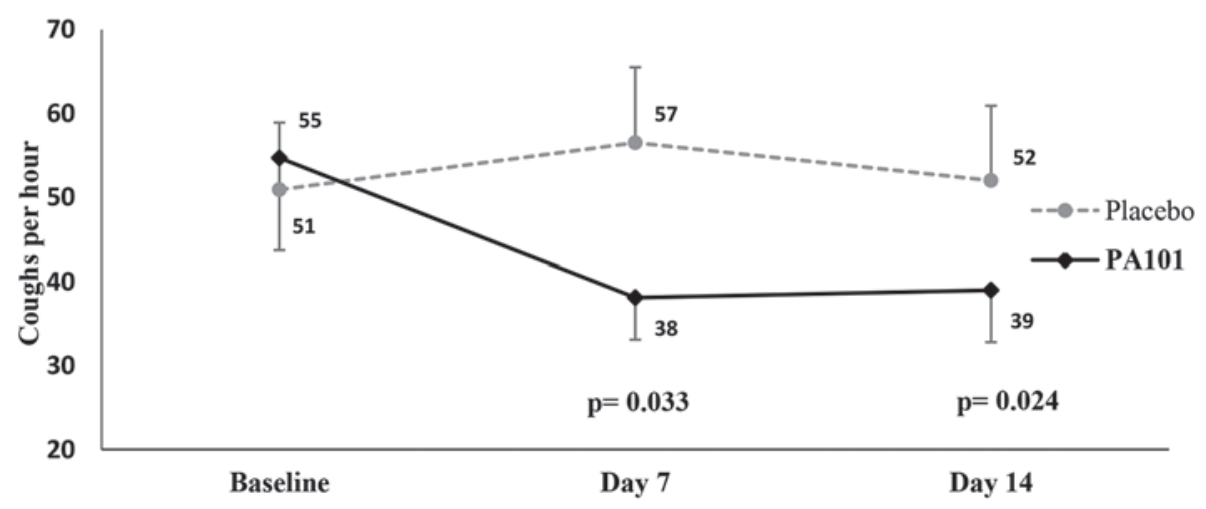

Abstract S34 Figure 1 Changes in daytime cough frequency in IPF cohort. 


\begin{tabular}{|c|c|c|c|c|}
\hline Efficacy Marker & $\begin{array}{c}\text { Baseline (off } \\
\text { morphine) }\end{array}$ & Morphine & Placebo & $\begin{array}{c}\text { P value (morphine v } \\
\text { placebo) }\end{array}$ \\
\hline $\begin{array}{c}\text { Daytime cough } \\
\text { frequency (c/hr) }\end{array}$ & 22.97 & 6.26 & 20.94 & $<0.05$ \\
\hline $\begin{array}{c}\text { Night time cough } \\
\text { frequency (c/hr) }\end{array}$ & 4.84 & 0.71 & 4.17 & $<0.05$ \\
\hline $\begin{array}{c}24 \text { hour recording } \\
\text { (c/hr) }\end{array}$ & 17.94 & 5.25 & 17.00 & $<0.05$ \\
\hline VAS Daytime (mm)* & 47.68 & 16.32 & 43.52 & $<0.05$ \\
\hline VAS night time (mm) & 25.00 & 3.00 & 19.00 & $<0.05$ \\
\hline CQLL* & 57.05 & 52.05 & 53.95 & $<0.05$ \\
\hline
\end{tabular}

Abstract S35 Figure 1 Median values for efficacy markers at baseline, on morphine and placebo *mean values.

scales (VAS)) and the cough quality of life questionnaire (CQLQ) . For analysis we ran generalised estimating equations to compare morphine and placebo, adjusting for baseline measures and assessing any influence of treatment, sequence or period.

Results Low dose morphine reduced objective cough frequency compared to placebo by $71.8 \%$ over 24 hours. Morphine significantly reduced cough frequency overnight as well as during the daytime $(p<0.05)$ and responses were independent of baseline cough frequency. All patient reported outcomes were consistent with this effect $(p<0.05)$. The treatment effect was not significantly influenced by period or sequence. Overall morphine was well tolerated. There was one serious adverse event, unrelated to the study treatment.

Conclusions This is the first study to demonstrate a substantial objective reduction in cough frequency with low dose morphine in a clinically responsive group. Importantly this was accompanied by improvements in patient reported outcomes despite the short treatment duration used. The data suggests this population would be suitable for further investigation of the mode of action of opioids in chronic cough.

\section{REFERENCE}

1. Morice AH et al. Opiate therapy in chronic cough. Am J Respir Crit Care Med 2007;175(4):312-5.

\section{S36 COUGH SUPPRESSION THERAPY IN SECONDARY CARE}

SJ Mohammed, J Steer, J Ellis, L Kellett, SM Parker. North Tyneside General Hospital, North Shields, UK

\subsection{6/thoraxinl-2017-210983.42}

Introduction Non pharmacological cough suppression therapy (CST) delivered by speech and language therapists is one of the few effective treatments available for patients suffering from a chronic refractory cough (CRC) although its use is not currently widespread. CST is a relatively new and developing area, our understanding of its mechanisms and the optimal treatment strategies are limited. We currently have no way of predicting response to therapy.

Method Retrospective review of records from patients undergoing CST in the period 2014-2017. Objective measures included quality of life (QOL) measured using the Leicester cough questionnaire (LCQ) and Laryngeal symptoms (Newcastle Laryngeal Hypersensitivity Questionnaire, LHQ).
Results 198 predominantly female (73.2\%) patients with chronic refractory cough underwent CST. Baseline/follow up LCQ and LHQ scores were available on 183/122 patients and $144 / 90$ patients respectively. Most had a cough of $>1$ year duration (85.9\%), impaired QOL (Baseline LCQ mean=11.6 (SD 3.52) and significant laryngeal symptoms (Baseline LHQ: mean 14.7 SD 3.08). The median number of CST sessions was 3. CST improved both QOL (Mean change in $\mathrm{LCQ}=4.97$ $\mathrm{SD}$ 3.81) and laryngeal symptoms (Mean change in $\mathrm{LHQ}=3.13 \mathrm{SD}$ 2.81). Most patients had a response greater than the minimally clinically important difference (MCID) for both LCQ (84.4\%) and LHQ (64.4\%) but a significant subgroup showed no improvement in objective markers or were worse after CST (15.6\% LCQ, 34.4\% LHQ). Sex, age, previous smoking status, duration of cough and baseline LHQ and LCQ did not predict non response to treatment.

Conclusions This is the largest series of patients undergoing CST published so far. Most patients show meaningful improvements in both symptoms and quality of life. Use of CST is not currently widespread and it is important that we improve access to this therapy. There is a significant subgroup of treatment non responders. Identifying those most likely to benefit will help us to personalise therapy for this challenging group of patients.

\section{Hot topics in home-based mechanical ventilation}

\section{S37 HOME MECHANICAL VENTILATION (HMV) AND HOME OXYGEN THERAPY (HOT) FOLLOWING AN ACUTE EXACERBATION OF COPD IN PATIENTS WITH PERSISTENT HYPERCAPNIA: RESULTS OF THE PER PROTOCOL ANALYSIS FROM THE HOT-HMV UK TRIAL}

${ }^{1}$ PB Murphy, ${ }^{1} \mathrm{G}$ Arbane, ${ }^{2} \mathrm{R}$ Phillips, ${ }^{1} \mathrm{~N}$ Hart. 'Guy's and St Thomas' NHS Foundation Trust, London, UK; ${ }^{2}$ King's College London, London, UK

\subsection{6/thoraxjnl-2017-210983.43}

Introduction Intention-to-treat analysis from the HOT-HMV UK trial showed an improvement in admission-free survival with the addition of home mechanical ventilation (HMV) to home oxygen therapy (HOT) in patients with persistent hypercapnia following an acute exacerbation of COPD 